\title{
25. STELLAR PHOTOMETRY AND POLARIMETRY (PHOTOMÉTRIE ET POLARIMÉTRIE STELLAIRES)
}

PRESIDENT: M. Golay.

VICE-PRESIDENT: A. Hiltner.

ORGANIZING COMMITTEE: A. W. J. Cousins, D. L. Crawford, T. Gehrels, J. A. Graham, A. R. Hyland, M. F. McCarthy, K. Osawa, V. Straižys.

\section{INTRODUCTION}

The Proceedings of the Conference on Electronography and Astronomical Applications held at the McDonald Observatory on March 11-12, 1974 and edited by G. L. Chincarini, P. Y. Griboval and H. J. Smith have marked the beginning of a new era, namely that of electronographic image devices.

Very interesting progress has been made then in instrumentation and reduction techniques. New photometric performances have been achieved by M. G. Walker, G. L. Chincarini, A. C. Hewitt, G. E. Kron, G. Wlérick, F. Lelièvre, H. Ables, G. Newell and E. O'Neil. For example, the electronographic observations of four clusters in the Magellanic Clouds have made possible the construction of colour-magnitude diagrams to much fainter limits than it had been possible using conventional photographic and photoelectric techniques (as faint as $V=22.5$ ) and this extension of the diagram to intrinsically fainter stars has provided new information regarding the age and chemical composition of clusters and their member stars.

Electronography has been successfully applied to surface photometry of globular clusters, galaxies, etc. Reduction techniques are able to reach internal precisions of 0.01 to 0.02 for the $V$ magnitude and 0.02 to 0.03 for $B-V$ with $14<V<17.5$.

Photometric data are used more and more to compare theoretical stellar atmosphere models with the observed spectral energy distributions. With theoretical models becoming more accurate, the photometric system ought to be better defined as well and better understood. We therefore recommend authors of papers containing photometric data to publish enough details concerning their observational procedures; i.e., the precise shape of the passbands, the equipment used, reduction procedures, list of standard stars used to establish colour equation, in order to enable readers to perceive clearly what has been done and thus permit them to use the given data suitably.

\section{REPORTS FROM MEMBERS}

\section{W. Becker (Basel Observatory)}

The photometry of fields in higher galactic latitudes is continued at the Basel Observatory. Plates taken with the 48-in. Palomar-Schmidt in the $R G U$ system are used. The following fields are observed: SA 82, 94, 107 and near to the following globular clusters: M 3, M 5, M 13, NGC 4147 and NGC 6171.

Steinlin and Buser (Astronomisches Institut Basel) are working on fundamental problems of multicolor photometry (see report of Commission 45).

\section{A. V. Landolt (Louisiana State University)}

Landolt's extension of the $U B V$ photometric system to fainter magnitude levels in the 24 celestial equatorial Selected Areas appeared in (10.113.087). The majority of the stars are in 
the range $10<V<13$; they cover a range of 2 mag. in $(B-V)$ and 3 magnitudes in $(U-B)$. Finding charts for each Selected Area, printed on one side only to facilitate ease of use at the telescope, are available from the author.

\section{K. Osawa (Tokyo Astronomical Observatory)}

A multi-channel photometer was installed at the Cassegrain focus of the $188-\mathrm{cm}$ telescope of the Okayama Astrophysical Observatory. At present, seven channels are available. Work for doubling the number of channels is in progress.

\section{Observatory of Belgium (Uccle)}

Denoyelle has established six $U B V$ photoelectric sequences in Vela, between $l=257^{\circ}$ and $l=281^{\circ}$, along the galactic equator. The photometric data are based on observations made with both the $50-\mathrm{cm}$ and $100-\mathrm{cm}$ telescopes of the European Southern Observatory.

The sequences were selected in view of future extensive photographic photometry on Schmidt plates covering the section of the southern Milky Way from $l=275^{\circ}$ to $284^{\circ}$. The limiting V-magnitude varies between 12.75 (Seq. III) and 16.11 (Seq. V).

The paper provides a series of charts for identification purposes.

\section{REFERENCE}

Denoyelle, J.: 1975, Astron. Astrophys. Suppl. 19, 45.

\section{A. Feinstein (Observatorio La Plata)}

$\mathrm{H} \alpha, \mathrm{H} \beta$ and $\mathrm{H} \gamma$ lines were measured photoelectrically with interference filters in a large number of stars (12.114.059).

About 100 Be stars were included with the aim to study the variation of the emission.

Also with a liquid nitrogen cooled $\mathrm{PbS}$ photoconductor it was begun to measure infrared magnitudes in the $J H K L$ system, of stars with suspected infrared excess.

H. Marraco started to measured polarization of some selected objects of the southern hemisphere.

\section{G. Chis (Observatoire de l'Université de Cluj-Napoca)}

A l'Observatoire de l'Université de Cluj-Napoca, on a effectué le passage du système photométrique instrumental au système standard $U B V$, nécessaire pour la réduction des observations photoélectriques (Chis and Pop, 1974). On poursuit l'étude de la variation de l'extinction atmosphérique dans les domaines $U B V$.

On déplace les instruments d'observation de l'ancienne place, qui se trouve en dedans de la ville, en dehors, à $8 \mathrm{~km}$ au sud, sur une colline couverte de forêts, et la détermination des nouvelles constantes photométriques est en voie d'exécution.

\section{REFERENCE}

Chis, D. and Pop, V.: 1974, 'Trecerea de la sistemul fotometric la sistemul standard $U B V$. Utilizarea datelor extinctiei atmosferice' (sous presse).

\section{A. G. D. Philip (Dudley Observatory, Albany)}

A conference, 'Multicolor Photometry and the Theoretical HR Diagram', was organized and held at the State University of New York at Albany in October 1974. All the major photometric systems were represented at the conference. 
Matlock has convolved the atmospheric models of Mihalas with the transmission curves of the $u v b y$ filters to yield theoretical four-color indices as functions of $\log g$ and $\theta_{\mathrm{e}}$. The predicted $\log g$ and $\theta_{\mathrm{e}}$ values are in agreement with those predicted by similar procedures by Breger, Kodeira, Kurucz, and Olson, reported at the conference.

Philip and Newell have established a temperature calibration, $(u-b)$, which works well for Population I and.II B stars.

Philip, Hauck, and Magnenat have presented a list of stars common to various photometric systems. Eighty-two stars (ranging from $\mathrm{O} 9$ to $\mathrm{K} \mathrm{5}$ ) are common to the five major systems, $U B V$, four-color, DDO, Vilnius, and Geneva systems.

Four-color photometry of blue horizontal-branch in globular clusters is continuing. These are indications that the morphology of the horizontal-branch is a function of the metal abundance of the cluster. Blue horizontal-branch stars in metal-poor clusters have four-color indices that agree with the models of Sweigart and Gross for stars with $Y=0.3, Z=10^{-3}$, Mass $=0.6 M_{\odot}$ and Core Mass $=0.475 M_{\odot}$.

Over 100 field horizontal-branch stars have been measured at the present time by Philip, J. Graham, J. Drilling, and P. W. Hill in the $u v b y$ system. The distribution of the field horizontal-branch stars in a los $g, \theta_{\mathrm{e}}$ diagram matches that of blue horizontal-branch stars in globular clusters.

Four-color observations of standard stars (9.113.011) of the new open cluster in line of sight with the large Magellanic Cloud (9.153.013) and in the globular clusters M 4 (9.154.014) have been published. Four-color photometry of some faint early-type stars in the direction of the Niagellanic Stream (11.159.004) indicates that some of these stars are of high luminosity. Radial velocity studies show that the stars are not field horizontal-branch stars. One interpretation is that these stars are members of the stream.

With C. L. Perry, of Louisiana State University, a review of four-color photometry from its beginning through 31 December 1975 is in preparation.

\section{J. Dachs (Astronomisches Institut Ruhr-Universität, Bochum)}

A photoelectric rapid-scanning single-channel spectrophotometer has been put into operation at the $61-\mathrm{cm}$ reflector of the University of Bochum installed at the European Southern Observatory, La Silla, Chile. The maximum resolution obtained with the scanner is $1 \AA$ in the second order of the grating $(3000-5000 \AA)$ and $2 \AA$ in the first order $(5000-8000 \AA)$. The instrument will be described in a forthcoming paper by Desjardins, Haupt, Maitzen, Rudolph, Schlosser and Schmidt-Kaler (1975, in preparation).

H. Tueg, Bochum, is at present comparing spectral energy distributions of southern and northern B- and A-type stars including $a$ Lyr with each other and with copper and platinum melting-point black-bodies of his own design (cf. Astron. Astrophys. 37, 249, 1974), using the Bochum scanner at La Silla and the Lowell Observatory scanner.

A system of standard stars for photoelectric $\mathrm{Ha}$ line photometry with narrow-band interference filters has been established by J. Dachs and Th. Schmidt-Kaler (Astron. Astrophys. Suppl. 21, 81, 1975). Two-dimensional calibration of Crawford's $\beta$ index of $\mathrm{H} \beta$ line photometry for early-type stars in terms of both spectral type and luminosity class has been performed by Th. Schmidt-Kaler and W. Klinkmann (1975, in preparation).

H. M. Maitzen, Bochum, has defined a degree of peculiarity for Ap stars determined from photoelectric narrow-band photometry only (Second European Regional Meeting in Astrono$m y$, in press).

\section{A. W. J. Cousins (Royal Observatory, South Africa)}

The SAAO is active in stellar photometry, mainly in the broad bands $(U, B, V, R$ and $I)$ and continuing the infrared photometry $(J, K$ and $L)$ begun by Dr. Glass, using more-or-less conventional techniques. The University of Cape Town Astronomy Department is more interested in high resolution photometry which includes an area scanner used to obtain $U B V$ photometry of close double stars. 
The activity of A. W. J. Cousins has been in connection with VRI photometry, setting up standards for future work (in the $\mathrm{E}$ and $\mathrm{F}$ regions, Magellanic Clouds, etc.) and intercomparing his photometry with earlier work at the longer wavelengths. The $R, I$ system used resembles Kron's and Eggen's.

Much of the observing at the Sutherland outstation has been variable stars and to provide sequences for photographic photometry, but most staff members and visiting astronomers have short projects of their own. Some photometry in the Strömgren bands and some photometry with a Spectracon image tube have been done.

\section{Breger (Department of Astronomy, University of Texas, Austin)}

\section{Calibration of $u v b y \beta$ Photometry}

The zero-points of the $u v b y$ system were determined from over 800 spectral scans of stars. For population I stars of spectral types B 0 to $\mathrm{G} 0$, to the $u v b y$ indices were calibrated in terms of $T_{\text {eff }}$ and $\log g$ by the use of model atmospheres (12.113.019). This calibration was also extended to the $\beta$ index (in 'Multicolor Photometry and the Theoretical H-R Diagram'). These calibrations are updated at present by using new ZAMS values being established by Crawford as well as measuring more line-blocking coefficients from coudé spectra.

\section{Spectrophotometric Scanner Catalog (unpublished)}

Over a thousand published and unpublished photoelectric scans of stars have been collected. These observations have all been transformed to a uniform absolute calibration of Vega. A uniform system relative to Vega has been defined from measurements by Bahner, Hayes, Oke and their co-workers. For a few other observers small systematic corrections have been applied to the measured fluxes to correct for systematic errors in the secondary standards. Since sets of $50 \AA$ scans can be converted to $u v b y$ indices to a precision of $\pm 0.015 \mathrm{mag}$. or better, we have looked for systematic Right Ascension errors in the scans. For some observers these Right Ascension effects are quite substantial. New secondary standards free of Right Ascension errors have been set up from the available data.

\section{Polarization of Young Stars in Dusty Regions (unpublished)}

Linear polarization of young stars near the Orion Nebulae has been measured. About $30 \%$ of the stars brighter than $V=15$ show significant polarization. The wavelength dependence of polarization divides the polarization into two groups: (i) stars with circumstellar shells. These stars show a very steep $p(\lambda)$ law, (ii) stars behind intracluster dust. This polarization follows the standard $p(\lambda)$ law established by Serkowski and others as well as a wide range of grain sizes (i.e. the wavelength of maximum polarization). A comparison with published as well as new infrared data obtained by Gehrz indicates a good relation between the value of $R$ (total to selective absorption) and the wavelength of maximum polarization. This Orion relation lies on the envelope of the scatter of a similar field star relation. This indicates that another physical factor besides grain size also determines the wavelength of maximum polarization $\left(\lambda_{\max }\right)$. We are presently attempting to study the effect of chemical abundance in the intracluster medium on the values of $R$ and $\lambda_{\max }$.

\section{R. King (Leuschner Observatory, Berkeley, Calif.)}

(1) I have been working on the color equations of various systems of photographic magnitudes. Most important, the standard photographic $B$ and $V$ systems (i.e., the standard platefilter combinations) appear to have appreciable color equations with respect to the photoelectrically defined $B$ and $V$. These are commonly neglected, and the consequences can be serious. For example a current paper (Faber, Burstein, Tinsley, and King, Astron. J., in press) shows 
that Weistrop's erroneous high density of $M$ dwarfs arose from just such an error in color equation. A second color-equation project is to study the relation between $R G U$ and $U B V$. This is a collaboration with the Basel group and is still incomplete but it is clear that the relationship for late-type stars is far from the color equations published by Steinlin for early-type stars.

(2) Ignorance of photographic color equations can easily arise from circular logic in deriving them. Observers have often looked at the residuals from an iris-to-magnitude calibration curve and found them satisfactorily small, not realizing that the residuals were small only because the curve was expressly drawn to fit the points. Only redundant points (i.e., several stars in a small range of magnitude) can give information about a color equation.

(3) In the course of a photometric study of SA 51, 57, and 68, I have had occasion to assemble photoelectric magnitudes from a number of sources and to combine them into standard sequences that are stronger than any published ones in those areas. The sequence for SA 57 includes some unpublished observations from several sources. A by-product of this work has been a redetermination of the color equation between the Stebbins-Whitford-Johnson $\mathrm{PV}$ and the standard $U B V$.

(4) On the Berkeley PDS computer-controlled microdensitometer, we have been experimenting with methods of stellar photometry through automatic analysis of photographic images. Even with simple reduction algorithms, the accuracy is better than that of an iris photometer. The present measuring rate is 3 stars per minute.

\section{H. Eelsalu (W. Struve Astrophysical Observatory, Tartu)}

H. Eeelsalu a consacré deux chapitres à la photométrie dans sa monographie (écrite en russe) Les Principes Statistiques de l'Astronomie Galactique Optique, Tartu, 1973-1975. Notamment le 9ème s'intitule 'Les Particularités de la Méthode Photographiques de l'Accumulation de l'Information', et le 12ème - 'La Notion du Système des Magnitudes Stellaires Photoélectriques'.

Il signale un article du Prof. V. Riives sur l'étude des images extrafocales (en russe, un résumé en anglais) 'On the Standardization of the Photographs of Comets' (10.031.051) ainsi que l'article de G. Kuzmin, T. Kübbar, V. Malyuto, 'Telescope and Photometer "Mizar", (12.032.047) qui a un Appendice: Le Schema d'Opération de la Lentille de Fabry pour le Télescope du Système Cassegrain.

\section{B. J. Bok (Steward Observatory, University of Arizona, Tucson)}

The present Report summarizes the work on photometric standard sequences done since 1973 by Ellis W. Miller and Carolyn C. McCarthy, directed by Bart J. Bok. The paper in which the new results are reported is published in Astron. J. 79, 1294, 1974; it is No. III in a series of papers by B. J. Bok, P. F. Bok and E. W. Miller. Seven new $U B V$ photoelectric sequences have been prepared for fields in Monoceros, Puppis and Vela, the magnitude limit of each sequence being $V=15.3$ or fainter. The magnitude range has been extended for three standard sequences by Bok, Bok and Miller (Astron. J. 77, 733, 1972), all of them located in Vela. With the new data, the total number of standard sequences has been brought to 33 , which means that the whole section of the Southern Milky Way between galactic longitudes $210^{\circ}$ and $335^{\circ}$ is now adequately covered with standard $U B V$ sequences $8<V<15$ on a uniform system. All of the recent observations were made at Cerro Tololo Interamerican Observatory. The research work was supported by a grant from the National Science Foundation. This may be a good occasion to note again that Argue, Bok and Miller have published a 'Catalogue of Photometric Sequences' plus one Supplement, work done under the auspices of Commission 25 of the International Astronomical Union.

\section{L. Straižys:}

On the Investigation in Photometry and Polarimetry in the Soviet Observatories

The activity of the Special Astrophysical Observatory of the U.S.S.R. Academy of Sciences 
was concentrated mainly on the development of the photoelectric instrumental equipment for detection of rapid fluctuations of brightness of astronomical objects. Such fluctuations were detected in SS Cyg, AX Mon and AG Peg (Alexeev et al., Izv. SAO 7 and 8, 1975; IAU Symp. 67, 1974). Another instrumental and computational complex was worked out for investigation of variations of brightness and polarization in the time scale up to $10^{-7} \mathrm{~s}$. Such ultrarapid variations of brightness were found to be absent in a number of stars observed, including white dwarfs DC, X-ray sources, and lacertides (Evseev et al., IAU Symp. 67, 1974).

In the Crimean observatory a method to determine, outside the atmosphere, magnitudes and colors of stars has been developed. It gives also the possibility to control currently the stability of instrumental photometric system and to determine the spectral response of the photometer (Nikonov, Izv. Crimean Obs. 54, 1975). TV system with image orthicon is used for routine photometry of faint stars in $B, V$ colors. The limiting measurable magnitude is $19 \mathrm{~m} .5$ in blue with $0.5 \mathrm{~m}$ Maksutov telescope with accuracy $\pm 0 \mathrm{~m} 2$. This TV system is being used for photometry of X-ray sources (Abramenko, Gollandskij, Prokofjeva). A special device allows to investigate from TV photographs rapid variations of brightness down to objects with periods of the order of few hundredths of second (11.141.306).

In the Sternberg Astronomical Institute of Moscow University the photoelectric UBV integral photometry of globular clusters both of our Galaxy and of M 31 was undertaken (Kukarkin, Šarov, Liutyj et al., 11.154.005, Letters to Soviet Astron. 1, No. 4, 3, 1975; Trudy Sternberg Inst. 47, 1976).

In the Astronomical Observatory of Leningrad University some infrared stars were observed photometrically in the system $U B V R I K$. The intrinsic polarization measurements were made for a number of IR stars, red variable stars, white dwarfs, symbiotic stars, X-ray sources, compact extragalactic objects etc. (Dombrovskij, Sulov, Khozov et al., 08.113,001; 08.131.046; $09.126 .004 ; 09.142 .149 ; 09.122 .142 ; 09.122 .143 ; 09.122 .144 ; 11.122 .098 ; 11.113 .036$; $11.122 .099 ; 11.131 .085 ; 11.142 .066 ; 09.141 .135 ; 11.158 .091)$.

The polarimetry of late-type irregular stars and of the stars of $\mathrm{R} \mathrm{CrB}$ type was made in Astronomical Observatory of Ukrainian Academy of Sciences near Kiev (Kolotilov, Orlov, Rodriguez, 10.122.076; IAU Symp. 67, 1975).

Some Soviet observatories were cooperating in photographic $U B V$ photometry of star fields containing open clusters or stellar rings (Kiev, Abastumani, Baldone, Sverdlovsk, Odessa Observatories, Sternberg Astr. Institute).

In Baldone Observatory of Latvian Academy of Sciences the $B V R$ photographic and photoelectric photometry of carbon stars in some galactic zones was continued (Alksnis, Alksne et al.).

The astronomers of Taškent Astronomical Institute started photometric observations in the new Mount Maidanak Observatory at $2760 \mathrm{~m}$ above sea level. This site is characterized by excellent observing conditions $-2000 \mathrm{~h}$ of observation per year, the star image sizes $\leqslant 0.25$ for $25 \%$ of observing time (Šev̌enko, Soviet Astron. 50, 632, 1973). The photoelectric photometry of $T$ Tauri type stars and photographic photometry of $T$ associations in UBVR system is being made (Sevčenko, Kardopolov).

The activity of the Vilnius Astronomical Observatory was concentrated on the further observations of stars in the optimum seven-color photometric system and on the revision of the ultraviolet magnitude of the system $U B V$.

The new method for classification of stars in temperatures and gravities was proposed by Straižys (12.113.040) using the reddening-free energy distribution curves obtained from multicolor observations. The method is suitable also to identify the stars of different peculiarities (subdwarfs, metal deficient giants, white dwarfs, Am stars, carbon, barium and zirconium stars, multiple stars etc.) by seven color photometry alone, without spectral observations and despite the presence of interstellar reddening.

The method is calibrated in temperatures and $\log g$ for all spectral types from $O$ to $M$ using model stellar atmospheres by Klinglesmith, Mihalas and Peytremann (Straizys, Rep. Dudley Obs. No. 9, 65, 1975).

The further catalogues of photoelectric photometry measured in the optimum photometric system were published (11.113.004; 11.113 .005 ; Bull. Vilnius Obs., No. 40, 1974). They in- 
clude 175 bright stars of different spectral types and 72 metal deficient stars. 26 Faint stars up to $V=15$ were observed around NML Cyg to obtain their two dimensional classification and to determine interstellar reddening (Zdanavicius and Kalytis, Bull. Vilnius Obs., No. 38, 1974). Bogdanovič and Alksnis (Baldone observatory) have used the Vilnius optimum system for photographic two-dimensional classification of 369 stars in the region of open cluster NGC 6871.

Sūdžius (Bull. Vilnius Obs. No. 39, 1974) has observed 68 O-type stars in the seven-color system to obtain interstellar reddening laws in different galactic longitudes. Combining the results by Whiteoak, Nandy and his own, he obtained two different laws of interstellar reddening - one for Cep-Per-Mon and another for Cyg. The laws include some fine structure pointed out in some investigations (Walker et al., Honeycutt, York, Hayes et al.).

The photoelectric standards of the Vilnius optimum system are being observed in SA 44, 49, $54,59,64$ at $-30^{\circ}$ and SA $92,98,104,110$ at $0^{\circ}$. In each SA about $20-30$ stars of various spectral and luminosity types in the magnitude range $7-9^{\mathrm{m}}$ are being measured with a $48 \mathrm{~cm}$ reflector placed in the Mount Maidanak Observatory in Middle Asia at $2760 \mathrm{~m}$ above sea level. The 63-cm reflector of new Moletai Observatory in Lithuania is used to investigate interstellar absorption in the direction of dark Taurus clouds, SA 64 in Cygnus and around Nova Cygni 1975 by photoelectric photometry of stars up to $12^{\mathrm{m}}$ in the Vilnius photometric system and in system $W B V R$.

The Nova Cygni 1975 itself discovered in Vilnius by amateur astronomer K. Černis is being observed starting August 30 both in Vilnius seven-color and in WBVR systems.

The revised ultraviolet magnitude of the system $U B V$ defined by Straižys (10.113.048) was tested observationally. The observations of 105 stars in the system $W B V R$ confirm the earlier theoretical conclusions that the revised magnitude $W$ with $\lambda_{0}=3475 \AA$ and $\Delta \lambda=510 \AA$ is suitable to replace the magnitude $U$ in the system $U B V$. The new magnitude $W$ has no shortcomings of the original $U$. The index $W-B$ is transformed outside the atmosphere with variable extinction coefficient dependent on spectral type, luminosity and interstellar reddening. The method of such a transformation was described by Zdanavičius (Bull. Vilnius Obs. No. 41,3, 1975). The standards of the revised system $W B V R$ are being observed by Vilnius Observatory $48 \mathrm{~cm}$ telescope placed in the Mount Maidanak Observatory.

\section{F. Rufener (Geneva Observatory)}

A compilation of the photoelectric measurements in the Geneva Observatory seven colour system $\left(U, B, V, B_{1}, B_{2}, V_{1}, G\right)$ is under publication. This new catalogue includes colours and $V$ magnitudes for 4670 stars and contains detailed discussions of the internal accuracy of the measurements.

Another catalogue is also under publication, giving, for all stars of the $U B V B_{1} B_{2} G$ catalogue, the parameters and indices used in the Geneva photometric system.

\section{G. Goy (Geneva Observatory)}

We are continuing our study which covers the general aspects of $O$ stars. The up-to-date third edition of the $O$ star Catalogue has already been published. It contains the description of 763 stars. A study (in press) shows that almost $100 \%$ of the $O$ type stars are either double or multiple and that their spectroscopical detection is very difficult. This latter fact leads us to suppose that the distances to these stars are generally under-estimated.

The microphotometer, designed for automatic measurement, is controlled by a computer and assisted by an operator. It is designed for the measurement of electrographic plates; special care has been taken to ease the control and positioning of the measured field.

The data stored on magnetic tape are analyzed later on.

We use a 32 K HP 2100 computer equipped with an ASR 33 board, a disc unit, a magnetic tape unit, a fast line printer, and a card reader and punch. A Tektronix video display equipped with a complete image storage system complements the system.

About 50 electrographic plates of clusters (NGC 7128, NGC 581 and M 67) in seven colours 
are being processed. The installation of a Kron camera for the new 1-m telescope at Gornergrat is nearing completion.

\section{A. Maeder (Geneva Observatory)}

Measurements and interpretation of several new kinds of variable stars of small amplitude is now in progress with G. Burki and F. Rufener. Particularly it appears that variability is connected with low rotational velocities for all B-type stars of class $V$ and IV and for A-type stars of class III. The phenomenon is not limited to the area of $\beta \mathrm{CMa}$ stars. Long series of measurements of B-F Supergiants in $h+\chi$ Persei clusters have been obtained and allow to find some relations between the amplitudes, the characteristic time of variations and the evolutionary status of these stars. The study of a variability appearing in some late F-subgiants is also in progress.

The accurate photometry of some old open star clusters has led to the confirmation of some new mechanisms in stellar evolution (over-shooting from convective cores).

The necessity of new data for some still older clusters appear to be urgent, because good evolutionary sequences can offer the possibility of testing some of the mechanisms proposed for testing the solar neutrinos problems. The realisation of these new observational tests are planned in common with Dr Crawford.

\section{Huguenin (Geneva Observatory)}

A new 8-band multipurpose $U V$ photometer has been launched on the stellar stabilized balloon platform of the Geneva Observatory. An advanced filtering technique has been used to achieve high peak transmission (30 to 50\%) and high rejection outside the bands $\left(10^{-4}\right.$ to $\left.10^{-5}\right)$. Each filter consists of a narrow-band multidielectric filter $(\Delta \lambda=4 \mathrm{~nm})$ and a four-reflection dichroic blocking filter. The filter-wheel holds six such filters, centered respectively at 205 , $216,273,295,307$ and $336 \mathrm{~nm}$ and two visible filters of the Geneva photometric system, $B 1$ and $V 1$.

The detector is a low noise bialkali photomultiplier, EMI $9789 \mathrm{QB}$, using single photoelectron counting. The system noise, including cosmic ray induced pulses at the altitude of $41 \mathrm{~km}$, is 15 counts per second. The pulse pair resolution is $52 \mathrm{~ns}$. B type stars with a $m_{\mathrm{v}}=6.7$ have been measured with a $15 \mathrm{~cm}$ Cassegrain objective.

\section{B. Hauck \\ (Institut d'Astronomie de l'Université de Lausanne et Observatoire de Genève)}

\section{Relations between Photometric Systems}

During the period considered, we have devoted a great part of the time to studying the relations between photometric systems. It is very important to derive these relations considering the various factors which may perturb such relations. In a first study (1) we derived, for the main-sequence stars, the relation between temperature parameters of some photometric systems (Geneva, $4 C-\beta$, Vilnius, six-color, UBVRI). This work was undertaken with the photometric catalogues compiled within the framework of the $C D S$ at Strasbourg and it constitutes the first step in a comparison between the different photometric systems. The next steps will be the effects of blanketing and luminosity on the relations derived above.

Based on the discussions at the Albany Colloquium, a choice of standard stars useful for calibration in the H-R diagram was made and photometric references given in (2).

\section{Photometric Catalogues}

In (3) we have discussed the conception of a stellar data centre. We have continued the compilation of photometric data. The list of catalogues on magnetic tape was published in (4) 
and (5). The following items were prepared during the period considered:

1. $4 C-\beta$ catalogue (6). A new version is in preparation.

2. A new catalogue of stellar $U B V$ photoelectric photometry (7). A new version is in preparation.

3. Catalogue of $U B V$ measurements and MK spectral types in open clusters (8). Data for 200 clusters.

4. $U B V$ Cape system in preparation by B. Nicolet.

5. DDO, Vilnius, 102.65.82 (Eggen) systems, see (5).

6. Preparation by P. Magnenat of a catalogue on magnetic tape indicating the photometric systems in which a star is recorded (at present approximately 35000 stars in 30 systems).

\section{REFERENCES}

1. Hauck, B. and Magnenat, P.: 1975, in A.G.D. Philip (ed.) Multicolor Photometry and the Theoretical HR Diagram (in press).

2. Philip, A. G. D., Hauck, B., and Magnenat, P.: 1975, in A. G. D. Philip (ed.), Multicolor Photometry and the Theoretical HR Diagram (in press).

3. Hauck, B.: 1974, Inf. Bull. Centre de Données Stellaires, No. 6, 1.

4. Hauck, B. and Jung, J.: 1974, Astron. Astrophys. Suppl. 16, 289.

5. Jung, J. and Guilbaut, M.: 1974, Inf. Bull. Centre de Données Stellaires, No. 7, 40.

6. Lindemann, E. and Hauck, B.: 1973, Astron. Astrophys. Suppl. 11, 119.

7. Mermilliod, J.-Cl.: 1973, Inf. Bull., Centre de Données Stellaires, No. 4, 20.

8. Mermilliod, J.-Cl.: 1975, in preparation.

\section{P. Mianes (Observatoire de Lyon)}

L'Observatoire de Lyon a axé ses recherches principalement sur la photométrie. Les différentes orientations sont les suivantes:

- Infrarouge (Mme Lunel, MM. Bergeat-Sibille), groupe équipé d'un photomètre infrarouge pour le domaine spectral $1-3.5 \mu$, étudie l'émission des enveloppes d'étoiles carbonées et le rougissement du rayonnement du gaz dans les régions $\mathrm{H}$ II compactes.

- Photométrie électronographique $U B V$ et étude morphologique de la partie interne du bras sud de M 33.

- Photométrie photographique en UBVRI (MM. Terzan, Rutilly) des amas galactiques et globulaires pour la détection et l'étude des variables (photographie classique et tube-image).

- Détermination des distances extragalactiques (M. Paturel) par l'utilisation des magnitudes et des diamètres photométriques des galaxies - Étude de la loi de Hubble.

- Photométrie photoélectrique (Mme Martel, M. Gravina) pour la polarisation des étoiles, des sources infrarouges, des condensations et des nébulosités incluses dans une dizaine de petites régions $\mathrm{H}$ II: Il a été observé des variations importantes de polarisation dans les quasars Ton 1542 et OJ 287 et rapides dans l'UV des étoiles Be.

- Photométrie du Grand Nuage de Magellan en $U B V$ et en rouge (Mlle Rousseau et M. Mianes) des supergéantes, pour l'étude de la structure et de la composition chimique du Nuage.

- Photométrie $U B V$ pour la recherche des variations de faibles amplitudes et à court terme des étoiles $B$ à enveloppe (Merlin).

- Un télescope Ritchey-Chrétien de $100 \mathrm{~cm}$ est en cours d'installation à la Station du Gornergrat (Suisse) à $3100 \mathrm{~m}$ d'altitude: cette implantation est le résultat d'une convention avec la Fondation du Jungfraujoch (collaboration INAG - Fondation - Genève - Lyon). La mise en service est prévue pour le printemps 1976.

\section{Photomètres}

Un photomètre infrarouge, et un photomètre pour la mesure des étoiles variables sont en cours de réalisation: Ils seront mis en service au cours de l'année 1976. 
- Un photocomparateur Blink a été réalisé. Il peut recevoir des clichés de $300 \times 300 \mathrm{~mm}$ et est entiérement digitalisé.

\section{Viton (Laboratoire d'Astronomie Spatiale, Marseille)}

\section{Expérience $D 2 B$}

Le satellite astronomique français D28 a été lancé avec succès le 27 septembre 1975.

Il emportait à son bord deux expériences de photométrie photoélectrique dans l'ultra-violet et une expérience plus technologique destinée au repérage de champ, mais devant apporter aussi des informations photométriques pour les étoiles les plus brillantes dans le proche UV. Les deux expériences principales, basées sur le principe du réseau objectif ont des bandes passantes d'allure gaussienne, résumées dans le tableau suivant:

\section{Bandes passantes des instruments $D 2 B$}

$\begin{array}{lrrrr}\text { Expérience } & \lambda 3090 & \lambda 2550 & \lambda 1600 & \lambda 792 \\ \text { Anti-solaire } & \Delta \lambda 310 & \Delta \lambda 320 & \Delta \lambda 300 & \Delta \lambda 70 \\ \text { Expérience } & & & & \\ \text { Lumière } & \lambda 3100 & \lambda 2200 & \lambda 1650 & \lambda 1216 \\ \text { Zodiacale } & \Delta \lambda 500 & \Delta \lambda 410 & \Delta \lambda 300 & \Delta \lambda 500\end{array}$

La magnitude 8 a été atteinte avec précision pour une étoile de type $\mathrm{B} 0$.

\section{Expérience à grand champ}

Destinées à la détection et à la photométrie stellaire et nébulaire dans l'ultra-violet, quatre caméras à grand champ ( $5000^{\circ}$ carrés) ont été lancées sur fusées-sondes de 1967 à 1972 , avec des bandes passantes larges ( 500 à $1000 \AA)$.

Deux tirs ont été réussis:

- en avril 1967 , avec 700 étoiles $O$ à $F$ détectées, dont 176 ont fourni des magnitudes à $2600 \AA$ avec un écart-type de 0.16 mag. par la classique méthode du photomètre à iris. Ces données ont permis une étude poussée de la situation des différentes classes de luminosité et des étoiles particulières par rapport à la séquence principale (publications en cours);

- en décembre 1972 , avec 1500 étoiles $O$ à $G$ détectées à 2600 et $3300 \AA$, dont la réduction des magnitudes est en cours.

\section{Expérience $S 183$}

Près de 4000 étoiles ont été enregistrées pendant les trois missions Skylab, à l'aide d'un photomètre de type Cassegrain $(f=74 \mathrm{~mm}$, ouverture $f / 2.8)$ la bande passante centrée à $2574 \AA$ (longueur d'onde moyenne) a une largeur moyenne de $358 \AA$. Le photomètre a un champ de $5^{\circ} \times 7^{\circ}$. La plupart des champs sont reportés le long de la Voie Lactée et comprennent divers objets tels que amas ouverts galaxies et nébuleuses. La réduction des mesures concerne le Grand Nuage de Magellan et $M 31$ et a donné lieu à deux publications en cours de préparation.

\section{Expérience Faust}

L'expérience Faust (télescope de focale $180 \mathrm{~mm}$, ouverture numérique $f / 1.12$, champ $5^{\circ}$, disposant d'intensificateurs d'images) a permis d'obtenir deux clichés de l'amas des Pléiades $(\lambda=2400 \AA$ et $\lambda=1600 \AA)$ et de détecter le flux anormalement intense de la galaxie $M 31$ à $1600 \AA$. 


\section{R. M. West (European Southern Observatory)}

The $u v b y$ and $\mathrm{H} \beta$ photometry has been made for over 1000 stars brighter than about 13.5 mag. and located generally in the Galactic polar regions. Spectral types have also been determined for many of the stars. These data constitute the initial phase of an extensive study of the kinematics of the Intermediate Population II stars.

\section{REPORT ON ESO-CHILE PHOTOMETRIC PROGRAMS}

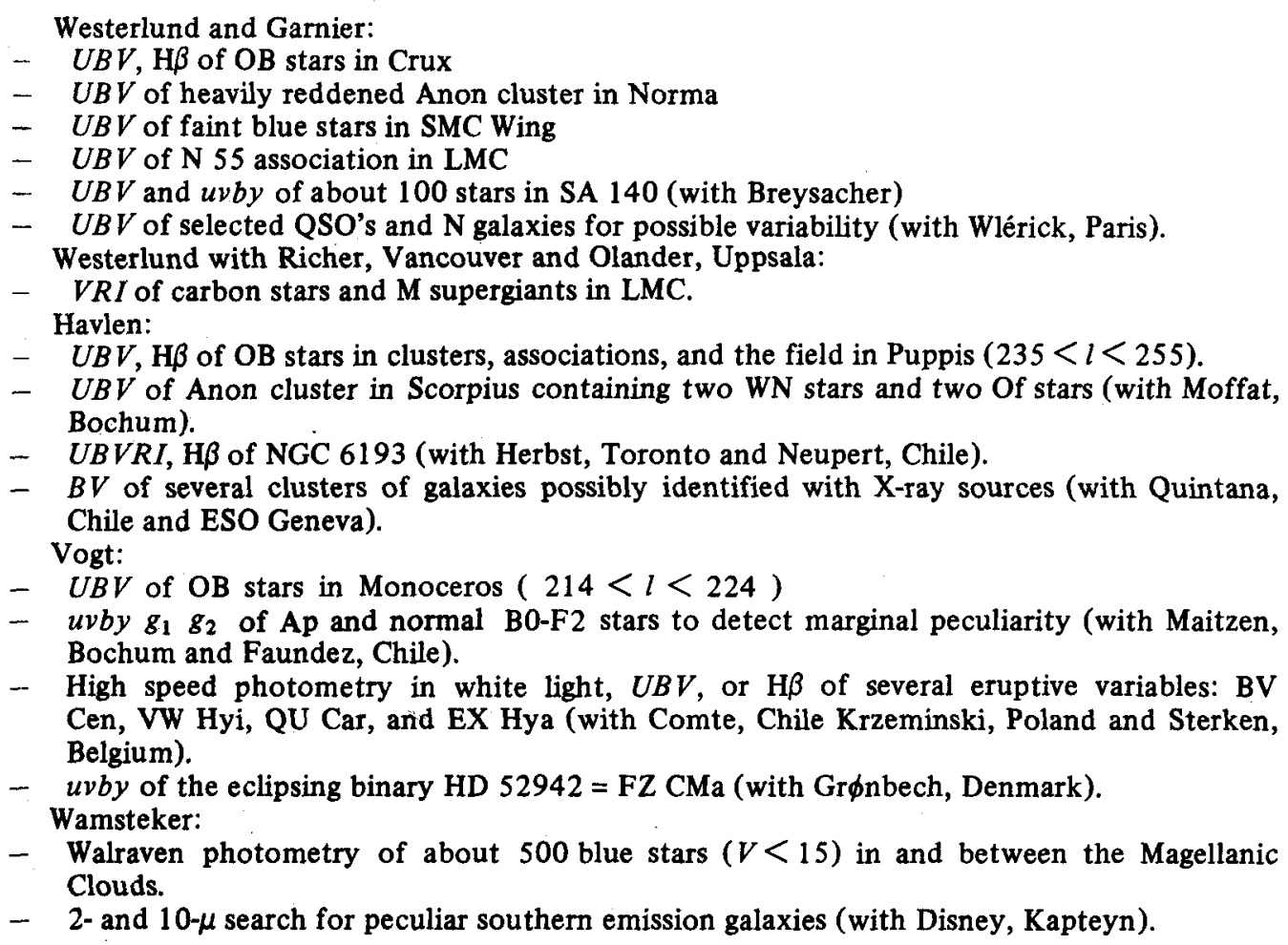

\section{POLARIMETRY}

(K. Serkowski)

The present summary of work on stellar polarimetry lists the review papers, the catalogues and lists of standard stars, and descriptions of new observing techniques. The papers containing the polarimetric observations for various types of stars and their discussions, numbering about 130 titles during the last three years, are discussed in the reports of the corresponding Commissions, particularly Commissions No. $27,34,36$, and 42.

\section{A. Review Articles}

The exhaustive reviews and bibliography of the entire field of astronomical polarimetry can be found in the book edited by Gehrels (1974). Problems of polarization by dust were reviewed by Aannestad and Purcell (10.131.066) and in the Proceedings of IAU Symp. 52 (10.012.022), intrinsic polarization of Be stars by Coyne (1976). Techniques of measuring polarization were reviewed by Serkowski (1974, and 12.034.100). 


\section{B. Catalogues and Standard Stars}

The proposed standard stars, either unpolarized or with well observed interstellar linear polarization, are listed by Serkowski (12.034.100 and 1974). Accurate measurements of linear polarization for stars in nearby open clusters, which may be useful as standards, were made by Markkanen (11.131.052, 12.131.072).

Catalogues of wavelengths $\lambda_{\max }$ of maximum interstellar polarization were published for several hundred stars by Coyne et al. (11.131.083) and by Serkowski et al. (1975). Measurements of visual or $2.2 \mu \mathrm{m}$ linear polarization for several hundred stars in dark cloud complexes Rho Oph, R CrA, NGC 1333, and Lynds 1630 were made by Vrba (1976) who found for a number of stars linear polarization considerably larger than any observed before. A survey of circular polarization for 84 stars was made by Stokes et al. $(11.131 .121)$.

\section{Observing Techniques}

The efficiency of wide-band observations of linear polarization was increased by eliminating the polarization of sky background (Piirola 10.034.013), by observing at many spectral regions simultaneously, and by using rapid modulation of light, thus saving the time which used to be spent on calibrations with insertable depolarizer. A ten-channel linear polarimeter in which the $U B V R I$ spectral regions are separated by dichroic filters, is described by Serkowski et al. (1975). A linear polarimeter with rapidly rotating superachromatic half-wave plate, operated by a minicomputer, is described by Frecker and Serkowski (1976) who use either photomultipliers or a Digicon photoelectron counting image tube as a detector; a transmission grating-prism forms a spectrum of $32 \AA$ resolution on Digicon photocathode, with polarization of sky background eliminated by using three focal plane diaphragms. Digicon is also used for linear and circular spectropolarimetry by Angel (12.126.024); similarly as in his very fruitfully used photomultiplier polarimeter (10.142.035), he used here a Pockels cell as a modulator. A large number of highly accurate measurements of stellar circular and linear polarization has been obtained with photoelastic polarimeters described by Kemp and Rudy (1975); an application of such an instrument to high-speed polarimetry of DQ Her is described by Kemp et al. (12.124.103). Still higher time resolution is obtained in optical polarimetry of the Crab pulsar by Cocke et al. (10.141.514) and Ferguson et al. (11.141.333). Stellar polarimeters with high spectral resolution were described by Borra and Landstreet (10.116.014 and 015), Fahlman and Walker (1975). Mavko et al. (11.131.042), Nordsieck (11.034.082), while wide-band polarimeters by Coulson et al. (11.034.043), Kinman and Mahaffey (11.034.085), Oshchepkov (11.117.018), Tinbergen (09.034.006), and Xanfomaliti and Dzhiapashvili (09.034.017).

Unfortunately no stellar ultraviolet polarimetry was made yet from any spacecraft. The only stellar polarimetry ever made at wavelengths shorter than $0.30 \mu \mathrm{m}$ is balloon polarimetry of two stars by Gehrels (11.131.084). Infrared stellar linear polarimetry was extended to wavelengths over $10 \mu \mathrm{m}$ by Dyck et al. $(10.131 .034,12.141 .420)$, and circular polarimetry to $3.5 \mu \mathrm{m}$ by Serkowski and Rieke (10.131.035).

Methods of producing scatter-free polaroids and plastic retardation plates and of making plastic achromatic retardation plates, potentially very useful for stellar polarimetry, were described by Title $(10.034 .107,1975)$. Transmission of grid polarizers was discussed by Stobbie and Dignam (1973). Polarization by coude mirrors was studied by Clarke (09.034.014).

\section{REFERENCES}

Coyne, G. V.: 1976, IAU Symp. 70.

Fahlman, G. G. and Walker, G. A. H.: 1975, Astrophys. J. 200, 22.

Frecker, J. E. and Serkowski, K.: 1976, Appl. Opt. 15 (in press).

Gehrels, T. (ed.): 1974, Planets, Stars and Nebulae Studied with Photopolarimetry, Univ. of Arizona Press.

Kemp, J. E. and Rudy, R. J.: 1975, Publ. Astron. Soc. Pacific 87, 301. 
Serkowski, K.: 1974, in N. Carleton (ed.), Methods of Experimental Physics, 12: 'Astrophysics, Part A: Optical and Infrared', Academic Press, New York-London, p. 361.

Serkowski, K., Mathewson, D. S., and Ford, V. L.: 1975, Astrophys. J. 196, 261.

Stobbie, R. W. and Dignam, M. J.: 1973, Appl. Opt. 12, 1390.

Title, A. M.: 1975, Appl. Opt. 14, 229.

Vrba, F.: 1976, Astrophys. J. (in preparation).

\section{Addendum}

A. T. Young (Texas A and M University) gives in the book Methods of Experimental Physics, Vol. 12, Astrophysics: Part A (Optical and Infrared), edited by N. P. Carleton (Academic Press, 1974), a review of the techniques of photometry very helpful to students. The book also contains chapters by D. W. Latham on Photographic emulsions and photometry. 